\title{
La complexification des connaissances et des dispositifs organisationnels et leur impact sur la fiabilité des opérations de l'aviation civile
}

\section{Knowledge and organizational process complexification and its impact on}

\section{the reliability of civil aviation's operations}

\author{
Pierre-Antoine Carpentier ${ }^{1}$, Mehran Ebrahimi ${ }^{2}$ \\ ${ }^{1}$ École des sciences de la gestion, Université du Québec à Montréal, Montréal, pierreacarpentier@gmail.com \\ ${ }^{2}$ Observatoire de l'Aéronautique et de l'Aviation Civile, Université du Québec à Montréal, Montréal, \\ ebrahimi.mehran@uqam.ca
}

RÉSUMÉ. Ce papier s'intéresse à la création des nouvelles connaissances dans les organisations de l'aviation civile. En nous basant sur des entrevues réalisées auprès de pilotes et de contrôleurs aériens, nous évaluons l'impact et les effets des interactions des professionnels de la sécurité aérienne sur la complexification des connaissances organisationnelles, sur les procédures implantées et sur la fiabilité qui en découle. Les résultats de nos analyses indiquent d'importantes lacunes communicationnelles qui peuvent mener à une réduction de la fiabilité des opérations de l'aviation civile, surtout lorsque les processus en cause sont liés à des tentatives de maximisation des profits.

ABSTRACT. This paper focuses on knowledge creation in civil aviation organizations. Based on interviews with pilots and air traffic controllers, we assess the impact and effects of the interactions of aviation security professionals on the complexification of organizational knowledge, and on established processes and their resulting reliability. Our findings indicate some significant gaps in organizational communication, which may lead to a reduction in the reliability of civil aviation operations, especially when the processes are linked to profit maximization.

MOTS-CLÉS. Organisations à haute fiabilité, trafic aérien, création de connaissances, dispositifs de gestion, aviation civile.

KEYWORDS. High-reliability organizations, air traffic control, knowledge creation, management processes, civil aviation.

\section{Introduction}

Le milieu de l'aviation civile est depuis quelques années soumis à des pressions économiques et opérationnelles sans précédent. Comme dans n'importe quel milieu, le secteur doit composer avec des impératifs de maximisation financière, mais à cela s'ajoute une augmentation fulgurante du trafic aérien. En effet, en 2018, c'est une progression de 6,1\% du nombre de passagers transportés qui a été observée, une augmentation qui s'ajoute au 7,9\% de l'année précédente [OAC 18]. L'Association du transport aérien international prévoit que, d'ici 2036, le nombre de voyageurs va doubler, atteignant 8,2 milliards de voyageurs par année [IAT 19].

Ces chiffres sont astronomiques et amènent déjà leur lot de défis dans les organisations impliquées dans la sécurité aérienne. Celles-ci opèrent selon les principes des organisations à haute fiabilité (high reliability organisations dans la littérature anglophone), où chaque erreur peut mener à des conséquences désastreuses, que ce soit en nombre de vies perdues, en termes de destruction de l'environnement ou pour l'entreprise elle-même. Ces organisations ont pour but la fiabilité et la sécurité maximale à tous les instants et sont, en dépit de la nature de leurs fonctions, parmi les plus efficientes et les plus performantes des organisations. En effet, en 2018, on parle d'un taux global de 1,35 accident par million de vols. Pour les 5 années précédentes, on parle d'un taux global de 1,79 [OAC 18]. Le secret de ce faible taux d'échec est une obsession pour la fiabilité et la sécurité de même qu'une batterie de procédures qui encadrent toutes les activités des organisations qui y sont liées. 
Le présent article s'intéresse en particulier à l'aviation et aux collaborations qui y prennent place, notamment entre les pilotes et les contrôleurs aériens. Ces acteurs sont au centre de l'écosystème de l'aviation civile et nous nous intéresserons ici aux procédures et dispositifs qui encadrent cette relation de même qu'aux divers mécanismes de création de connaissances qui font partie de leur quotidien.

\section{Littérature}

\subsection{Fiabilité}

D'abord, situons ces interactions. Comme mentionné plus tôt, on parle ici d'organisations qui ont comme but premier la fiabilité et la sécurité des opérations. Ces organisations ont également des caractéristiques précises qui les rendent reconnaissables, ou qui les démarquent des autres. Perrow (1984) [PER 84]., un pionnier du domaine, décrit celles-ci en parlant d'organisations complexes où l'interdépendance des activités peut mener à des suites d'opérations qui ne permettent pas beaucoup de temps de réaction ni d'adaptabilité aux employés qui se retrouvent inévitablement impliqués dans des incidents. Roberts (1990) [ROB 90]. s'inscrit dans la même veine en décrivant les organisations à haute fiabilité selon deux grandes caractéristiques ; leur fonctionnement à travers des technologies avancées et l'interdépendance qui relie les opérations de l'organisation, en plus d'une capacité à la fiabilité au quotidien.

Weick définit la fiabilité comme suit: «the unusual capacity to produce collective outcomes of a certain minimum quality repeatedly» (2008). Donc, une capacité unique à obtenir une performance sans failles de façon constante, ininterrompue. Morel (2014) [MOR 14]. décrit de son côté une fiabilité qui se traduit par des décisions et des actions conformes aux buts fixés, tout en étant une façon d'éviter les erreurs. Lekka (2011) [LEK 11]. évoque des organisations qui sont capables de maintenir des performances presque sans erreurs malgré le fait qu'elles opèrent dans des conditions dangereuses où les conséquences des erreurs peuvent être catastrophiques. C'est cette recherche constante de fiabilité qui est au cœur des organisations dites à haute fiabilité (dont l'aviation fait partie) et c'est ce qui oriente tous leurs processus ainsi que toutes leurs décisions. Cette préoccupation de tous les instants assure des conduites qu'on juge appropriées et surtout prévisibles à tous les niveaux organisationnels, du bas de l'échelle hiérarchique jusqu'aux sommets décisionnels les plus élevés [WEI 08].

Schulman (2008) [SCH 08] établit par ailleurs 2 modèles généraux de la fiabilité, celui de la Résilience et celui de la Prévention. Si le premier accepte l'inévitabilité des erreurs et des dysfonctionnements en tentant de limiter les dégâts après que ceux-ci soient arrivés, le second modèle se base sur une analyse de tous les processus en temps réel pour tenter de détecter les anomalies et prévenir les éventuels incidents. L'industrie de l'aviation civile se situe évidemment du côté de la prévention, ou du moins, on ose l'espérer.

Ainsi, les organisations qui entrent dans cette catégorie de fiabilité et de performance optimale doivent pouvoir être au courant de tout ce qui se passe à tous les instants, un concept qu'on peut traduire comme étant une conscience situationnelle (situational awareness) [END 01]. Durso (2004) [DUR 04] la définit comme étant la compréhension d'un opérateur de l'évolution de l'environnement où il effectue sa tâche. Weick évoque un état mental, le mindfulness, ou vigilance constante en français, pour décrire cet état d'esprit qui permettra éventuellement d'éviter les événements non désirables avant que ceux-ci ne se présentent (2008). Voici un schéma qui le résume bien : 


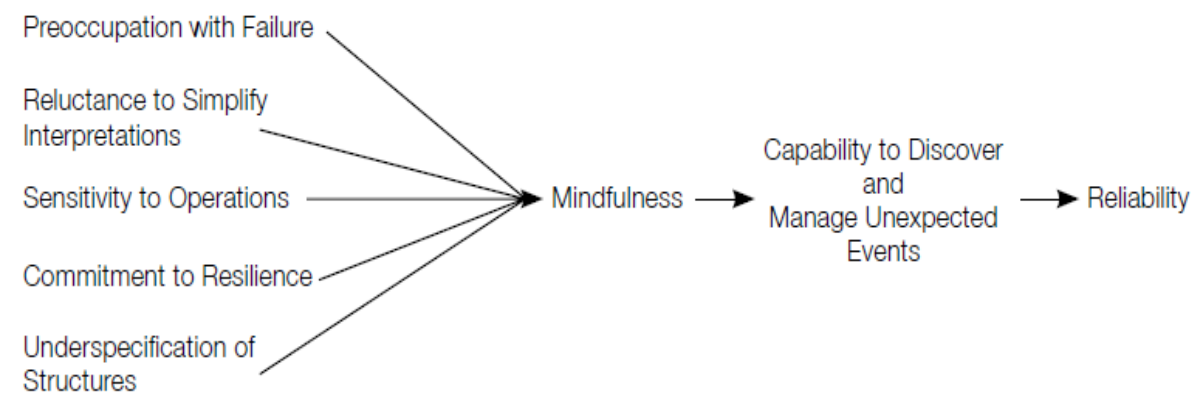

Figure 1. Les 5 processus essentiels à la gestion de la fiabilité (Weick, (2008), figure 1, p.37)

Grâce à ces 5 processus mis en place par les organisations à haute fiabilité, on arrive alors à détecter et augmenter ce sentiment de vigilance constante. Le premier processus est la préoccupation à l'échec (p.51), où les auteurs évoquent une attention particulière à tout ce qui peut constituer un écart à la normalité. Un souci des détails minutieux est à l'œuvre et on s'intéresse également aux near misses, ou presque échecs, un concept qu'abordent par ailleurs en profondeur Dillon et Tinsley (2008) [DIL 08]. Avec cette préoccupation à l'échec, on examine tous les événements qui ont dévié à la normalité, y compris ces événements qui n'ont pas mené à un incident, mais où il s'en est fallu de peu. Le second processus qui est discuté par l'équipe de Weick est la réticence à la simplification (p.41). On veut ici éviter les simplifications cognitives qu'on a tendance à faire pour simplifier notre environnement, qu'on insère dans des catégories de pensées précises. On se met alors dans un état de questionnement et de requestionnement perpétuel en ce qui concerne les procédures et les interprétations ; un travail éreintant, mais nécessaire à l'atteinte d'une performance stable et continue. Le troisième processus mis de l'avant est la sensibilité aux opérations (p.43). Ce dernier fait référence à un état mental qui vise à se faire un portrait global de toutes les données opérationnelles et cela de façon simultanée. On veut ainsi éviter de se faire prendre par surprise par les événements. Cela implique également une projection dans le futur. Cette projection dans le futur est nécessaire à une prise de décision éclairée et compréhensive qui atténuera les risques.

Le quatrième élément qui va définir les organisations à haute fiabilité est l'engagement à la résilience, qui indique que les employés doivent réagir rapidement avec les ressources dont ils disposent au moment où l'erreur se produit. L'organisation doit dans ce cas être formée et être prête lorsqu'une telle éventualité survient. Ces organisations acceptent selon Weick l'inévitabilité des erreurs et se préparent en fonction de ce postulat. Les organisations qui cherchent la fiabilité tentent par ailleurs également de développer des compétences en improvisation, pour ne pas se sentir désemparées lorsque la situation l'exige, tout en ne se fiant pas uniquement aux connaissances issues des événements passés (p.46).

Le cinquième processus s'appelle la sous-spécification des structures et se veut être une réponse aux organisations rigides qui propagent sans le savoir les défaillances à cause de règles qui ne sont pas contestées ou revues une fois qu'elles sont établies (p.48). Les erreurs y sont donc en quelque sorte amplifiées par un système ordonné et bien rodé. De plus, lorsque les erreurs viennent des hauts sommets hiérarchiques, elles sont moins souvent contestées et se répandront d'autant plus vite. Ainsi, dans les organisations à haute fiabilité, les structures hiérarchiques et décisionnelles se modulent selon les problèmes rencontrés. En fonction de ces derniers, les décisions finales ne sont pas nécessairement attribuées à ceux qui se situent au sommet de la pyramide décisionnelle, mais bien à ceux qui ont le plus de connaissances sur l'enjeu en question [MOR 14].

Ainsi, comme illustré dans la figure 1, ces 5 processus, une fois combinés, permettent une meilleure détection des conflits potentiels ainsi que des réactions adéquates et précises lorsque des incidents se présentent. Ces petites actions du quotidien forment un filet de vigilance constante qui va venir filtrer 
les événements, signifiants ou insignifiants. Nous verrons plus tard si ces principes sont appliqués au sein des relations de travail des contrôleurs aériens et des pilotes de ligne.

\subsection{Dispositifs}

Les principes énoncés dans la section précédente, bien qu'ils peuvent sembler intuitifs dans les circonstances, demandent une grande implication organisationnelle. Cette implication prend, la plupart du temps, la forme de dispositifs, de procédures que l'organisation met en place pour répondre aux demandes de son environnement. Voici ce qu'en disent les pionniers du domaine de la haute fiabilité. LaPorte (1998) [LAP 98] explique les dispositifs comme étant une génération de nouvelles connaissances qui est bâtie par une combinaison de vieilles connaissances et d'expériences confrontées à des nouvelles réalités. On les incorpore alors à l'ensemble de l'organisation sous la forme de dispositifs. Roberts (1990) [ROB 90], en contexte d'organisations à haute fiabilité, parle de rôles spécifiques donnés aux employés au sein de séquences invariables pour atteindre des buts fixés. Dujarier (2017) [DUJ 17] définit quant à elle le dispositif ainsi : «le mot s'apparente au planning, à l'organisation détaillée de l'action avant qu'elle ait lieu et déterminée loin d'elle » (p.17). Donc on a également ici une conception du dispositif comme étant un outil de gestion qui sert à guider et surtout prédire l'action des travailleurs. Chiapello (2013) [CHI 13] parle de son côté de formation de sens commun à travers ces outils de gestion, qui sont à la fois fonctionnels, structurels et processuels.

Dujarier (2017) [DUJ 17] décrit 3 sortes de dispositifs : de finalité, de procédés et d'enrôlement. Le dispositif de finalité établit les objectifs organisationnels quantifiés à atteindre et surtout les critères par lesquels le jugement de performance se fera. Les dispositifs de procédés sont complémentaires aux précédents, car ils comprennent la façon ou les outils qui doivent être utilisés pour atteindre les objectifs quantifiables. Ces dispositifs de procédés qui guident les actions des employés viennent également avec une temporalité précise, avec un rythme qui viendra délimiter les étapes de la journée du travailleur. Ainsi, cela vient bouleverser le rythme naturel ou improvisé qu'une journée peut prendre ; si une tâche prend plus de temps que prévu, cela sera noté et vu sous un jour négatif tout en enlevant de la marge de manœuvre pour accomplir la tâche ou l'action suivante. Donc, pour que tout se passe rondement, ces dispositifs de procédés doivent être utilisés dans les mêmes conditions que celles imaginées par leurs concepteurs. Enfin, il y a également les dispositifs d'enrôlement, qui sont en place pour que les employés coopèrent de façon uniforme avec la mise en place des procédés expliqués préalablement. Ce type de dispositifs passe par la communication interne des organisations et vise à convaincre les travailleurs des bienfaits de ces outils de gestion.

En contexte de haute fiabilité, on parle de processus routiniers, parfois répétitifs, qui servent également à détecter les anomalies. On s'assure ainsi qu'un élément qui n'est pas à sa place est automatiquement pris en considération [ROC 87]. On peut également voir des routines opérationnalisées sous la forme de checklists, notamment dans le milieu médical et dans celui de l'aviation [MOR 12]. Milosevic (2018) décrit des routines où les individus ont déjà rencontré des événements et peuvent utiliser leurs connaissances pour les gérer, mais aussi des non-routines qui nécessitent la création de nouvelles connaissances.

On parle aussi de redondance, une stratégie utilisée pour rendre fiables des systèmes qui sont intrinsèquement imparfaits [SAG 04] comme le facteur humain. On parle ici d'équipes qui emploient par exemple plus que le strict minimum d'employés dans le bus d'avoir des ressources disponibles et une meilleure adaptabilité en cas d'incident. On met en place certaines unités de rechange, au cas où la première unité est dans l'incapacité d'accomplir sa tâche lors d'un événement quelconque. Un peu à la manière des copilotes qui doivent faire du monitorage (dire à voix haute toutes les actions à faire de la part du pilote dans le bon ordre) lors des manœuvres qui ne sont pas prises en charge par le pilote automatique [MOR 12]. Rochlin (1998) [ROC 98] rapportait des procédures semblables dans la marine américaine, où les unités opèrent en deçà de leurs capacités pour que si l'une d'entre elles se bute à un problème ou devient dysfonctionnelle, ses tâches puissent être attribuées à une autre unité sans qu'elle 
soit débordée. Ces mesures ont pour but de réduire le stress qui est mis sur chacune des unités de l'organisation par la complexité et l'interdépendance de ses activités, un stress que Perrow (1984) [PER 84] nomme tight coupling dans sa théorie des accidents normaux.

Pour parler de la place de l'homme dans tout cela, Dejours (2014) [DEJ 14] décrit deux approches : on peut soit approcher le facteur humain en se disant qu'il faut limiter les défaillances humaines, ou encore on peut considérer l'humain comme une ressource précieuse qu'il faut mobiliser, développer et gérer. L'approche de défaillance humaine a comme préoccupation principale la sécurité et tentera de prédire toutes les situations de travail dans leurs intégralités. Grâce à cette connaissance des processus et de leurs débouchés, on sera en mesure d'établir des normes et des techniques strictes qui assureront selon ses concepteurs la sécurité des opérations. C'est la vision qu'applique le milieu des compagnies aériennes, qui gèrent les pilotes de ligne. L'approche des ressources place de son côté la priorité comme étant la qualité, la sécurité étant une conséquence qui découlera normalement de la qualité des opérations. Selon cette deuxième approche, on ne peut pas tout prescrire, on doit laisser une certaine place aux événements qu'on ne peut pas prévoir, à l'accidentel. L'individu s'inscrit ici dans un contexte socio-économique précis et interagit avec cet environnement, ce qui change inévitablement le cours des événements.

C'est ce décalage entre le réel et le prescrit qui différencie les deux approches, un concept que l'auteur situe au cœur de sa position sur le facteur humain dans l'organisation. Par le terme « réel », Dejours entend cette expérience subjective du travail vécue au quotidien par les employés et par l'organisation en général. Ce terme se veut être un rassemblement de tout ce qui ne peut être prévu, ou même décrit, qui ne peut entrer dans la logique de planification extensive valorisée par l'approche de la défaillance. Il décrit d'ailleurs dans un autre ouvrage le décalage entre le réel et le prescrit dans les évaluations que les organisations mettent en place pour noter le travail accompli, en disant qu'on ne peut accéder au subjectif des travailleurs de cette façon [DEJ 16]. Dujarier (2017) [DUJ 17] remarque sensiblement les mêmes choses en évoquant des dispositifs qui sont créés loin des opérateurs et sans leur participation. Chiapello (2013) [CHI 13] affirme que les fonctions prévues pour l'outil de gestion ne peuvent pas couvrir toutes ses fonctions possibles et ne peuvent anticiper parfaitement ses effets. Kloman (2001) [KLO 01] affirme que chaque décision ou procédure va avoir des conséquences qui sont intrinsèquement impossibles à anticiper, peu importe comment elles ont été conçues. L'utilisation des dispositifs, malgré le fait qu'elle soit bien intentionnée, peut en ce sens provoquer à son tour des incidents en raison de cet écart qui existe entre ce qu'on veut que les outils accomplissent et la réalité du quotidien de ceux qui les utilisent.

\subsection{Création de connaissances}

Pour créer des dispositifs efficaces, on se doit de les baser sur des connaissances organisationnelles pouvant être appliquées aux situations prévues à cet effet [NON 95]. Ikujiro Nonaka est ici une source inépuisable d'informations pertinentes dans le champ de la création de connaissances organisationnelles et c'est cette orientation théorique qui sera décrite dans la partie suivante pour y décrire les notions impliquées.

Nonaka et Takeuchi séparent d'abord la connaissance en deux parties, la connaissance tacite et la connaissance explicite. Les savoirs explicites peuvent être articulés en mots, dans un langage qui est accessible à celui qui transmet le savoir et à celui qui le reçoit. Les connaissances tacites sont au contraire plus difficiles à communiquer, car elles proviennent de l'expérience subjective et corporelle des travailleurs. De par leur nature subjective, ces connaissances sont liées avec le système de valeur de l'individu qui les possède, de même que ses croyances personnelles et la perspective unique qu'il aura par rapport à cette connaissance. Selon les auteurs, c'est le type de connaissance qui contient l'information critique en ce qui concerne les savoirs organisationnels, car elle renferme toute l'expérience accumulée par le travailleur (p.10). 
Les auteurs avancent par ailleurs qu'il y a deux dimensions présentes dans la connaissance tacite : technique et cognitive. La première dimension contient les talents et habiletés précises qui concernent le savoir-faire des individus dans l'accomplissement de leurs tâches. La seconde, cognitive, concerne notre vision du travail lui-même, la façon dont on le conçoit de même que les séquences attendues dans le futur (p.26). Nonaka et Takeuchi affirment que ces deux connaissances (tacites et explicites) sont constamment en relation et que c'est justement cette interaction qui va créer les connaissances à l'ensemble de l'organisation. On commence à partir des connaissances tacites du travailleur, qui le communiquera au groupe et ce groupe l'étendra ensuite à l'organisation.

La littérature offre d'autres visions de la connaissance qui ressemble à cette séparation taciteexplicite. Giddens (1984) [GID 84] affirme de son côté dans la théorie de la Structuration qu'il y a deux niveaux de conscience dans nos activités quotidiennes ; la conscience pratique et la conscience discursive. La conscience discursive nous donne des informations rationalisées pour guider nos actions de façon consciente et se base sur les informations explicites que notre expérience sociale nous a amenés à acquérir. La conscience pratique se base plutôt sur un niveau de connaissances auxquelles on ne pense pas vraiment et qu'on ne théorise pas ; on a ici le penchant tacite de cette théorie des connaissances. Kakihara et Sorensen (2002) [KAH 02] séparent plutôt la connaissance en 2 catégories : représentationnelle et anti-représentationnelle. La première voit le monde préétabli selon les cognitions et l'intelligence humaines et la seconde catégorie est le résultat d'interprétations, d'actes mentaux relationnels et d'interactions dynamiques entre le subjectif et l'objectif.

En ce qui concerne la création de connaissances, Nonaka et Toyama (2005) [NON 05] la considèrent à travers une interaction dynamique entre la subjectivité et l'objectivité, où les pensées et actions des individus sont synthétisées au sein de leurs relations avec leurs collègues à l'intérieur des frontières organisationnelles. Les auteurs avancent que sans la subjectivité des travailleurs et le contexte précis dans lequel cette subjectivité prend place, les connaissances ne peuvent exister ni prendre leur sens. Ils accordent également une place importante aux contradictions: " the theory of the knowledge-creating firm views a firm as an entity to create knowledge actively by synthesizing contradictions »(p.433). La création de connaissances organisationnelles est dans cette optique une connaissance tacite d'un employé qui est externalisée dans une connaissance objective qui est partagée et synthétisée par d'autres. Ces nouvelles connaissances sont utilisées et enrichies par les nouveaux utilisateurs, qui en feront des vérités subjectives à leur tour. Bohm (2004) affirme également que c'est dans ces dialogues interrelationnels que des sens communs organisationnels peuvent émerger.

Nonaka et Toyama (2003) [NON 03] amènent par ailleurs la notion du Bà pour expliquer la création des connaissances dans l'organisation. Ce mot japonais, qui veut dire « espace » en français, décrit un lieu où les dialogues constructifs prennent place et où les individus peuvent mettre en pratique les éléments de visions et d'objectifs de l'organisation. Les auteurs le définissent comme étant un «shared context in motion, in which knowledge is shared, created, and utilized »(p.6). Dans ces Bà, les contextes se rencontrent pour former un sens commun à travers les interactions des participants ; c'est un endroit où le subjectif est partagé, compris et synthétisé pour devenir une connaissance commune. Les particularités et les ambiguités des connaissances subjectives sont, grâce à ces interactions dynamiques, synthétisées grâce au dialogue. On part de la connaissance initiale, la thèse, qui est confrontée à une information contradictoire qui la rend incomplète, et on arrive à la conciliation des deux avec la connaissance complexifiée, la synthèse (Nonaka et Takeuchi, 2004) [NON 04]. Les auteurs affirment par contre que les contextes ne peuvent être identiques entre les individus, car ils ont une expérience et des points de vue qui sont différents les uns des autres, et c'est justement ce qui viendra enrichir le partage d'information. Nonaka et Takeuchi (2004) [NON 04] avancent justement que cette synthèse de connaissances sera d'autant plus riche si elle provient d'informations variées et même contradictoires. Nonaka et Van Krogh (2006) [NON 06] évoquent par contre le fait qu'en raison de ces vécus différents, la justification sociale des connaissances individuelles est difficile et fragile et n'est pas toujours synonyme de synthèse produisant de nouvelles connaissances. 
Au niveau de la créativité, Nonaka et Toyama (2002) [NON 02] affirment que ces environnements de travail doivent permettre une rétroaction constante qui remettra en question les acquis et qui offre des opportunités de création de nouvelles connaissances améliorées, synthétisées par la suite. Selon un cycle qu'ils appellent Kata, on doit apprendre, briser et créer à nouveau dans un cycle de connaissances dynamique et rétroactif. Les nouvelles connaissances ainsi créées doivent par ailleurs être intégrées avec succès au système de connaissances organisationnel en place [NON 06].

Dans le milieu de la sécurité aérienne, on peut inférer que c'est particulièrement difficile de créer de nouvelles connaissances et de les implanter dans l'organisation. En effet, les organisations à haute fiabilité se trouvent dans une case à part, ne pouvant vraisemblablement se permettre d'avoir des échecs à partir desquels on peut voir que les procédures ont mené à un échec. Ceux-ci menant à des catastrophes humaines et institutionnelles désastreuses, on se doit de trouver des alternatives pour construire la connaissance et rendre compte des défaillances dans les dispositifs. Dans un environnement aussi complexe, on ne peut non plus se fier à ses expériences passées pour établir des nouvelles procédures et connaissances, parce que celles-ci peuvent se montrer désuètes face aux défis qui se renouvèlent chaque jour. Nonaka et Van Krogh (2006) [NON 06] affirment que les organisations accumulent du capital de connaissance qui est issu de leurs succès passés, mais qui peut brimer leur adaptation aux nouvelles demandes de leur environnement. Ils affirment : "In the face of intensifying competition, market changes and technological disruption, the organization clings to its knowledge assets that have produced success in the business system layer (which) focuses more on applying existing knowledge assets than on generating its capacity for creativity »(p.1198). Ainsi, ces organisations se fient à leurs expériences passées pour s'adapter à leurs défis actuels, même si le contexte a changé. Nonaka et Toyama (2007) [NON 07] ajoutent que les problèmes surgissent chaque jour et sont différents des pronostics qui tentaient de les anticiper, ce qui forcera les employés à s'adapter, à modifier leurs stratégies dans la pratique. Ce qui permettra à certaines organisations de se démarquer sera alors leur capacité à s'adapter au changement perpétuel dans la pratique plutôt que de tenter d'analyser la situation pour en faire une entité finie. Pour ce faire, Nonaka et Toyama proposent le concept de phronesis, qui décrit des connaissances tacites de grande qualité qui sont acquises à partir d'expériences pratiques des individus et qui permettent de prendre des décisions autant prudentes qu'éclairées. Ces décisions sont également situées, guidées par les besoins contextuels spécifiques à chaque situation, et sont en continuité avec les valeurs et les objectifs de l'organisation.

\section{Problématique de recherche}

Il est important de noter que dans le milieu de l'aviation civile, et dans probablement bien des organisations à haute fiabilité, les connaissances et les dispositifs sont intimement liés. En effet, la connaissance est opérationnalisée sous la forme de dispositifs, pour guider les lignes d'action en fonction des connaissances que l'entreprise a à sa disposition. Lorsque les connaissances sont insuffisantes face à des nouvelles réalités rencontrées dans l'exercice des fonctions quotidiennes de l'organisation, celle-ci fournira des nouveaux dispositifs ou procédures pour y remédier. Ces nouveaux outils ne seront pas tout le temps efficaces ni complets, mais les mécanismes de la création de connaissance y sont quand même présents.

Notre projet de recherche se penche donc sur l'interaction entre ces trois domaines de la littérature ; la connaissance organisationnelle, les dispositifs et le milieu de la haute fiabilité. Plus précisément, à la création de nouvelles connaissances, qui sont acquises et implantées par le biais des dispositifs et des procédures dans des organisations qui ont comme but premier la fiabilité et une performance sans faille de ses opérations. Dans le cas qui nous intéresse, l'aviation civile, l'accent a été placé sur le métier de contrôleur aérien et celui de pilote de ligne. Ces deux professions ont leurs tâches respectives, mais doivent collaborer sur une base quotidienne en ayant un but commun : la sécurité aérienne. 
La question est ici de savoir comment leurs connaissances respectives s'articulent et entrent en relation au sein de ces interactions. Ces travailleurs effectuent leurs tâches en se basant d'une part sur leurs connaissances personnelles acquises grâce à leur expérience et leur formation et d'autre part sur les dispositifs et autres guides mis en place par leur organisation. Ces connaissances personnelles, comme il a été mentionné dans la section précédente, doivent être partagées et remises en question pour qu'elles soient complexifiées. La question de recherche est alors de savoir si et comment le contexte de l'aviation civile met en place ce genre d'interactions et de va-et-vient entre les connaissances pour augmenter la performance et la fiabilité de ses activités. Si c'est le cas, quels sont alors les mécanismes et les conditions qui sont en place et qui permettent cette articulation des connaissances ? Si au contraire ce contexte n'est pas propice au partage et à la création de connaissance, pourquoi ?

\section{Cadre conceptuel}

Pour analyser les données de notre projet de recherche, nous avons décidé d'utiliser le cadre théorique mis de l'avant par D.W. Holford [HOL 09, HOL 10]. Ce dernier se penche sur la relation entre la connaissance et le risque, affirmant qu'une intégration réussie par le dialogue de plusieurs types et sources de connaissances peut mener à une atténuation des situations à risque ou des conséquences indésirables. Voici le schéma (Figure 2) qui résume les relations entre les divers concepts, qui seront expliqués plus bas :

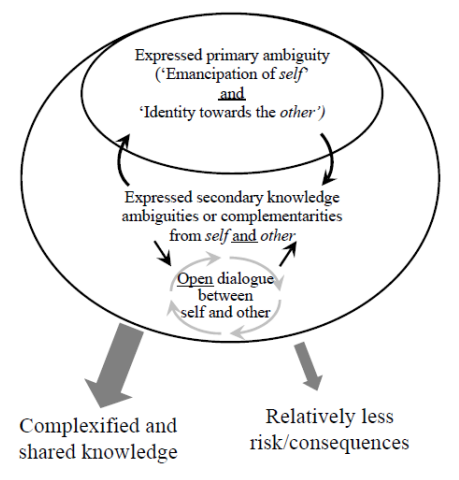

(a)

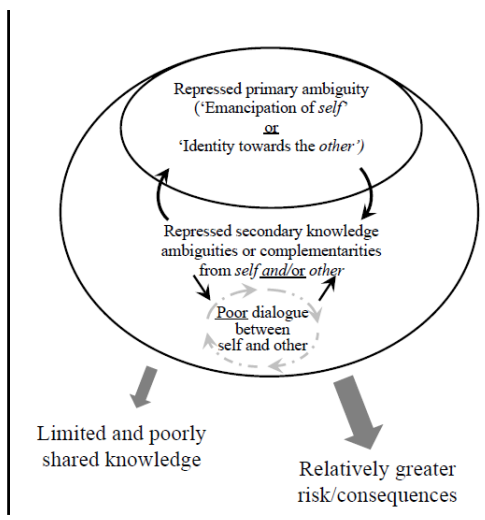

(b)

Figure 2. Modèle de la relation dialectique des ambiguïtés primaires et secondaires menant à la complexification des connaissances (Holford, 2010, figure 8, p.154).

Holford affirme que, pour obtenir une conversation dialogique constructive où la connaissance peut être échangée et bonifiée, les différents partis impliqués doivent faire ce qu'il appelle la prise de perspective et le don de perspective (perspective taking/giving, p.142) On prend ainsi le point de vue d'autrui en considération avant de former ou de modifier le nôtre, pour par la suite le donner à notre tour. Ces principes contribueront à la résolution de l'ambiguïté primaire. Celle-ci fait référence au désir qu'ont tous les humains de s'exprimer et de faire valoir leurs opinions alors qu'ils sont en même temps confrontés au désir d'écouter, de s'identifier aux autres et de se faire valider par eux. De l'autre côté, on doit également retrouver une écoute et un respect de l'autre et de ses perspectives pour que soit résolue cette ambivalence. C'est dans cette ambiguïté que prennent racine les relations de collaboration et d'interaction des individus dans une organisation (p.148). Sans cette ambiguïté primaire, la complexification des connaissances ne peut avoir lieu.

Après avoir acquis ce désir d'aller vers l'autre et de contester les acquis, on peut passer au second niveau : les ambiguïtés de connaissances secondaires. Celles-ci sont semblables à la synthèse dont parlaient Nonaka et Takeuchi (1995) [NON 95] : on y trouve une connaissance (thèse), qui se verra confrontée à une nouvelle information (antithèse) et le produit de cette interaction dialogique sera la connaissance complexifiée (synthèse). Cette résolution d'ambiguïté peut prendre de multiples formes, comme la transition de connaissances tacites à explicites par exemple. Les ambivalences et les 
ambiguïtés sous-jacentes à toute connaissance sont selon Holford ce qui permet, lorsqu'elles sont exprimées, d'approfondir et de complexifier les connaissances [WEI 01, BEC 01]. Ces ambiguités sont résolues au sein de relations dialogiques formelles et informelles, où on effectue des va-et-vient incessants entre les multiples sources de connaissance. Les connaissances obtenues deviennent ainsi des intégrations dynamiques de la collaboration de chacun, où tous les participants prêtent une partie de leurs acquis et de leurs expériences subjectives.

Grâce à ces dialogues constructifs, l'organisation peut s'attendre à moins de situations indésirables, à moins de risque de conséquences néfastes en raison de cette examination en profondeur des connaissances et de ses débouchés. Les risques sont induits lorsque les perspectives individuelles ne s'accordent pas avec les autres et ne sont pas examinées ou même formulées. Holford parle de risque comme étant un potentiel pour des conséquences néfastes, que celles-ci soient financières, sécuritaires ou environnementales. Ces catégories de risques sont inter reliées, inter causales et dialectiques (p.131). Pour Beck (2001) [BEC 01], le risque est un manque ou un trou dans les connaissances étant causé par des simplifications excessives de phénomènes intrinsèquement inter reliés et inter causaux dans des organisations complexes. Lawrence (1986) [LAW 86] évoque de son côté un phénomène mesurable en termes de probabilité et de sévérité d'un effet négatif.

Lorsque le dialogue ne s'installe pas entre l'individu et les autres, les connaissances restent stagnantes et ne s'enrichissent pas de la perspective des autres. L'organisation ne profite ainsi pas de l'expérience et des intuitions souvent précieuses de ses employés. Les dialogues en place ne sont donc pas constructifs et peuvent être mécaniques, forcés et ne permettent pas l'émancipation de soi pour le bien des connaissances organisationnelles.

\section{Méthodologie}

En ce qui concerne nos données, nous avons choisi d'utiliser une banque de données secondaire appartenant à l'Observatoire international de l'aviation civile et de l'aéronautique. Nous y avons sélectionné un total de 13 entrevues comprenant des discussions avec des pilotes de ligne et des contrôleurs aériens. Nous avons décidé d'arrêter à 13 en raison d'une saturation théorique des propos obtenus ; nous avons obtenu à 13 entrevues une redondance suffisante à nos objectifs de recherche. Les entrevues ont eu lieu entre juin 2018 et mai 2019 et ont une durée d'en moyenne une heure trente minutes. Les participants proviennent d'organisations diverses et de nationalités diverses, n'ayant en commun que les professions qu'ils exercent. Les entrevues totalisent près de 22 heures de discussions portant sur leurs expériences de travail et leurs relations de collaboration au sein de l'aviation civile. On parle ici de plus de 300 pages de verbatim qui ont été réalisées et analysées.

Dans ces entrevues, les pilotes et les contrôleurs aériens étaient questionnés sur la nature de leurs relations avec leurs collègues de travail, sur l'emprise des dispositifs dans leur quotidien, sur la complexité des opérations et sur la création de nouvelles connaissances, entre autres. Les relations de travail pouvaient traiter des dyades suivantes: Contrôleur aérien \& Pilote, Contrôleur aérien \& Organisation, Pilote \& Copilote et Pilote \& Organisation. Il s'agissait d'entrevues semi-directives où les participants répondaient aux questions posées tout en ayant une grande marge de manœuvre pour développer plus en profondeur les concepts abordés [AKT 87]. Le but est ici de laisser la chance aux répondants de mentionner des éléments qui ne sont pas nécessairement prévus dans le questionnaire.

Nous avons utilisé une approche qualitative, puisque nous cherchons à comprendre les interactions entre les sujets et comprendre le sens des implications qui en découlent [MIL 91, FOR 06]. L'important était ici d'aborder des phénomènes sociaux et organisationnels en mettant un accent sur leur signification plutôt que leur quantité [ANA 07]. La démarche y est également abductive [HAL 19], étant donné que nous faisons des aller-retour entre les données du terrain et la littérature existante sur le sujet, qui est en constante évolution [ANA 07]. Au niveau épistémologique, ce projet de recherche adopte une posture constructiviste [PER 03], c'est-à-dire que notre démarche de 
compréhension va de pair avec la construction des réalités sociales des acteurs, en gardant en tête la relativité des contextes et la subjectivité des interviewés [BER 96]. Selon la posture du constructivisme, « le monde social est fait d'interprétations qui se construisent grâce aux interactions entre acteurs, dans des contextes toujours particuliers. Les individus créent leur environnement par leur pensée et leurs actions, guidés par leurs finalités » [PER 03, p.24].

\section{Analyse de données}

Pour analyser ces données, nous avons d'abord retranscrit les entrevues dans leur intégralité. Par la suite, nous nous sommes intéressés à la présence ou non des thèmes et concepts pertinents dans notre contenu, étant donné que nous faisions une analyse de contenu qualitatif [AKT 87]. Le but premier est de faire émerger des retranscriptions les items et concepts pertinents à notre étude.

Nous avons ainsi employé les recommandations de Miles et Huberman (1991) [MIL 91] et effectué une analyse en 4 étapes ; la conceptualisation, la découverte des catégories, l'étiquetage des catégories et le développement des catégories. Le tout se fait évidemment manuellement et en gardant en tête les théories et connaissances évoquées dans la revue de littérature.

Toutes ces étapes ont été réalisées avec l'application NVivo, un programme d'analyse de données qualitatives. Ces analyses ont été faites dans le programme à l'aide d'une codification des données qualitatives issues des transcriptions auditives réalisées. Les étapes de découverte, d'étiquetage et de développement des catégories ont été faites à l'aide de nœuds primaires, dans lesquels nous avons développé des réseaux de nœuds sous la forme de sous-catégories. Le tableau 1 comprend les résultats de cette analyse de données.

La phase de conceptualisation comprend la recherche d'éléments qui apparaissent à répétition. Dans le document de retranscription, nous avons donc annoté les éléments qui revenaient plusieurs fois, dans le but de les dénombrer et de les situer plus tard par rapport à l'ensemble des items annotés. Ainsi, une grande quantité d'éléments jugés à priori pertinents ont été notés et seulement ceux qui étaient en lien avec le sujet ou qui sont d'une importance contextuelle importante ont été retenus. Ensuite, pour la phase de découverte de catégories, le but est de regrouper les concepts qui ont été notés dans la première phase pour en faire des catégories. Les éléments qui étaient trop marginaux n'ont pas été regroupés, l'objectif étant de simplifier les données recueillies. Nous nous sommes retrouvés avec deux grands regroupements primaires : les interactions positives et négatives dans le cadre des fonctions officielles des participants. Nous en avons dénoté 241 au total. En effet, les interviewés parlaient beaucoup de leurs relations avec les nombreuses parties prenantes de l'écosystème de l'aviation pour parler de leurs expériences subjectives de travail.

Par la suite, pour l'étiquetage des catégories et pour développer davantage ces grands regroupements, nous nous sommes basés sur les éléments que nous avons retrouvés dans la littérature pour classer les concepts. Nous avons donc regroupé les 241 situations-clés en 3 sous-catégories : création de connaissances, les dispositifs organisationnels et les impératifs de fiabilité et de performance. Les commentaires ont ainsi été classés selon leur concordance à l'un de nos trois piliers de littérature. Ensuite, pour le développement des catégories, nous avons séparé les situations-clés selon leurs propriétés et leurs dimensions pour former des sous-catégories. Dans le but d'analyser ces données en fonction de la littérature et de ce que le terrain a révélé, nous avons créé les sous-catégories suivantes pour les articuler en fonction du modèle de Holford (2010) [HOL 10] : Ambiguité primaire résolue, ambiguïté secondaire résolue, ambiguïté primaire non-résolue, ambiguïté secondaire nonrésolue et conséquences indésirables. Nous avons ainsi pu obtenir des données structurées et quantifiées qui nous indiquaient la fréquence relative des occurrences de réussites ou d'échec de complexification des connaissances dans la relation entre les pilotes, les contrôleurs aériens et leurs organisations. 


\begin{tabular}{|c|c|c|c|c|c|}
\hline Candidats & $\begin{array}{l}\text { A.P. non- } \\
\text { résolue }\end{array}$ & $\begin{array}{l}\text { A.S. non- } \\
\text { résolue }\end{array}$ & A.P. résolue & A.S. résolue & $\begin{array}{l}\text { Conséquences / } \\
\text { Risques }\end{array}$ \\
\hline 1-Hernie & 16 & 8 & 2 & & \\
\hline 2-Felix & 4 & 2 & 5 & 4 & \\
\hline 3-Florent & 12 & 7 & 4 & 2 & 2 \\
\hline 4-Guy & 7 & 2 & 1 & & 3 \\
\hline 5-Georges & 9 & 2 & 3 & 5 & 6 \\
\hline 6-Alex & 6 & 3 & 5 & 2 & \\
\hline 7-Eddy & 4 & 4 & & 3 & 4 \\
\hline 8-Etienne & 13 & 2 & 6 & 2 & 3 \\
\hline 9-Fred & 2 & 3 & 7 & 1 & 1 \\
\hline 10-Luc & 4 & 5 & 1 & 5 & 3 \\
\hline 11-Sandy & 3 & 4 & & 4 & 2 \\
\hline $12-\mathrm{Scott}$ & 5 & 3 & 3 & 7 & \\
\hline 13-Roland & 4 & 7 & 2 & & 2 \\
\hline TOTAUX & 89 & 52 & 39 & 35 & 26 \\
\hline
\end{tabular}

Tableau 1. Fréquences des commentaires-clés des participants en fonction des sous-catégories communicationnelles ${ }^{1}$

\section{Résultats}

\subsection{Articulation des concepts de Holford}

La section qui suit sert à démontrer que les interactions que nous avons retrouvées au fil des conversations avec les contrôleurs aériens et les pilotes reflètent la façon dont les savoirs sont véhiculés dans l'aviation civile. Pour donner un sens à nos données, nous articulons ainsi les situations et les commentaires mentionnés par les participants à l'aide du modèle de Holford. Les relations et les va-et-vient entre les différents acteurs par rapport à la construction de la connaissance sont cruciaux à l'atteinte de la fiabilité et ces mouvements s'illustrent très bien avec le modèle dialogique de la figure 2. Nous allons d'abord expliquer ce que chaque colonne du tableau 1 comprend en fournissant des exemples qui sont représentatifs de chacune d'entre elles.

\subsubsection{Ambiguïté primaire non-résolue}

Nous pouvons ainsi voir que ce sont les ambiguïtés primaires non-résolues qui sont les plus souvent décrites par les pilotes et les contrôleurs aériens, de près du double de cas relevés. Regardons quelques exemples concrets de ce type de situation.

Hernie : «Parce que souvent, il y a des pilotes qui disent : ah non je ne demanderai pas ça, je ne dirai pas que ça va trop vite et que j'ai besoin de ralentir. Ils ont peur. »

On voit ici un pilote, Hernie (nom de code) qui a peur de s'exprimer en raison des procédures de réprimandes mises en place dans la compagnie aérienne. Le problème, dans ce cas précis, est qu'il est

\footnotetext{
${ }^{1}$ Lorsqu'on parle d'ambiguité primaire non-résolue (A.P. non-résolue), cela signifie que les concepts évoqués plus tôt d'émancipation de soi et d'identité envers les autres ne sont pas présents. Ainsi, l'individu n'a pas le désir de s'exprimer ni d'écouter ce que l'autre a à lui dire, ce qui ne permet pas de résoudre l'ambiguité primaire. La prise de perspective et le don de perspective ne sont ainsi pas présents et ne peuvent mener à une conversation constructive. L'ambiguité secondaire non-résolue (A.S. non-résolue) évoque de son côté une situation où les connaissances sensibles, possiblement contradictoires et ambivalentes ne sont pas discutées et donc ne peuvent compléter le cycle de complexification des connaissances organisationnelles. Selon les concepts évoqués par Nonaka et Takeuchi (1995), on peut dire que l'antithèse n'est pas opposée à la thèse pour former la synthèse. Les termes ambiguités primaires résolues (A.P. résolue) et secondaires résolues (A.S. résolue) signifient que lors d'une situation donnée, les conditions nécessaires à la complexification sont réunies, avec leurs significations respectives. La colonne Conséquences / Risques comprend par ailleurs les commentaires des sujets où ils reconnaissaient voir le lien direct entre des conséquences indésirables concrètes et des défaillances au niveau de la communication des connaissances dans leurs activités quotidiennes.
} 
très difficile pour un pilote de se faire donner une restriction d'altitude et de vitesse en même temps. On peut difficilement descendre et décélérer, en d'autres termes, surtout lorsque l'avion en question est un jet. L'ambiguïté primaire n'est ici pas résolue parce que le pilote ne veut pas donner sa perspective sur l'état risqué de sa situation. Il ne cherche pas non plus à savoir ce que l'autre, dans ce cas-ci la compagnie et les contrôleurs aériens, ont à dire, même si en fin de compte, ça pourrait l'aider d'en parler. On obtient alors une situation potentiellement plus risquée en raison du manque de communication, car le pilote se sent forcé de faire quelque chose qu'il sait être plus dangereux.

Georges : «C'est souvent la peur de, dans les mains de qui va tomber l'information et qui va l'utiliser contre nous. On ne partage pas l'information auprès des pilotes, qui sont la dernière ligne de gestion et de protection d'une opération complexe. »

Ici Georges se plaint du manque de communications organisationnelles post-incident. En effet, plusieurs pilotes ont noté qu'après un incident qui implique par exemple des aéronefs qui passent près d'entrer en collision à cause de réglementations particulières propres à certains aéroports, les compagnies aériennes ne partagent pas nécessairement l'information critique qui a causé ou qui aurait pu causer l'incident. Le pilote en question, Georges, pense ici que l'organisation ne partage pas cette information de peur d'être prise en défaut, de peur de se faire poursuivre. Nous avons donc ici encore accès à une ambiguïté primaire non-résolue dans le sens où l'organisation augmente la chance qu'un événement problématique survienne parce qu'elle n'entame pas de conversation avec ses pilotes sur ce sujet critique. Le don de perspective de la part de l'organisation n'est évidemment pas présent dans cette situation.

Luc : « Ils ont atterri derrière un Luftanza. Quand Luftanza a atterri, il y avait beaucoup d'eau sur la piste et Luftanza avait dit que le breaking action, le rapport de freinage, était vraiment nul. Fait que le contrôleur de la tour, il l'a dit à (compagnie) qui était derrière, et le pilote de (compagnie) a dit, ce n'est pas grave, nous autres on continue. Le contrôleur a dit ok, va atterrir piste 33 gauche. Ils ont crashé en fin de piste. »

On voit ici un exemple évident d'une communication où l'ambiguïté primaire n'est pas résolue. La relation pilote-contrôleur aérien est structurée de la façon suivante ; le contrôleur aérien émet des recommandations aux pilotes et ceux-ci décident ultimement ce qu'ils font avec leur avion, car ils sont les seuls responsables de la sécurité de leur appareil. Ils devront se justifier à leur compagnie s'ils ne suivent pas les recommandations des contrôleurs, mais sur le coup, ce sont eux qui prennent la décision finale du chemin de l'avion. Nous avons alors dans cet exemple le contrôleur aérien qui donne sa perspective alors que le pilote ne la reçoit pas (perspective taking), annulant ainsi la tentative d'identification envers l'autre.

\subsubsection{Ambiguïté secondaire non-résolue}

La seule autre catégorie d'événements-clés qui sont unanimement relevés par les participants dans les entrevues est les ambiguïtés secondaires non-résolues (A.S. non-résolue dans le tableau 1). Donc on évoque dans les extraits suivants des cas où la conversation est établie entre soi et l'autre, mais où les connaissances partagées sont refoulées, forcées ou limitées [HOL 10, p.153].

Florent : «L'apprentissage devient un petit peu secondaire, surtout dans nos programmes de formation. Actuellement, c'est plus pour cocher des cases : ça il l'a vu, il l'a vu, ça il l'a vu. Plutôt que de vraiment former...»

Il est dans cet extrait question des formations qui sont imposées aux pilotes chaque année pour s'assurer qu'ils restent au bon niveau lorsqu'ils sont aux commandes des appareils. On voit ici que les connaissances n'y sont pas examinées en profondeur, mais plutôt uniquement vérifiées pour satisfaire les exigences de qualification des pilotes. Il n'y a ainsi pas de remise en question des connaissances, pas de visions qu'on oppose pour former ces pilotes. Le dialogue est forcé et la formation n'est donnée 
que pour satisfaire des formalités de nature bureaucratique. On peut logiquement en déduire, selon le modèle de Horford, que ces formations bâclées ne présentent pas une plateforme adéquate à la résolution des ambiguités secondaires et mènent directement à des risques augmentés d'incident et de conséquences indésirables.

Guy: «Tous les incidents qu'il y avait eu sur la ligne, ils les publiaient sous forme papier, cachetée, et lorsqu'on allait aux opérations, on pouvait en prendre une (revue). Et pendant le vol, on pouvait lire les 30,40 incidents qu'il y avait eu. Et ils ont arrêté cette pratique. Maintenant nous avons une publication qui publie des incidents choisis, que la compagnie a choisi de partager. Mais nous n'avons pas accès à tous les incidents comme on avait autrefois. »

Nous avons dans cet extrait une ambiguïté secondaire non-résolue, car l'organisation choisit de cacher certaines informations qui pourraient être cruciales à la sécurité de ses opérations. Certaines connaissances sont ainsi refoulées par l'organisation et ne sont pas soumises à la contestation, à la révision de l'autre participant de la conversation, les pilotes. Des potentiels renforcements à la connaissance sont ainsi consciemment ignorés et la connaissance obtenue est incomplète, même si le potentiel aux apprentissages est clairement présent.

\subsubsection{Ambiguïté primaire résolue}

La troisième colonne comprend les situations où les ambiguïtés primaires ont été résolues et ont permis de pousser la conversation plus loin, de partager des perspectives. Le dialogue ouvert est, rappelons-nous, la première marche qui mène à des conversions et des transferts de connaissance.

Etienne : «Aussi, quand tu te qualifies, une fois par mois, la première année, y'a quelqu'un que toi tu choisis qui t'accompagnes. [...] Fait que mettons, nous on travaille dans une sous-unité, puis que moi j'aime comment tu travailles, bien je vais dire ok, lui, mettezmoi avec lui une fois par mois. »

Nous avons ici l'exemple d'un dispositif où c'est l'organisation elle-même qui met en place un procédé qui favorise le dialogue ouvert. L'individu qui a moins d'expérience est ainsi pris en charge par un vétéran et ce dernier a l'opportunité et la responsabilité de partager ses connaissances sous la forme d'un mentorat formel. C'est ici l'organisation qui est ouverte à la communication et qui respecte le fait que celui qui débute le métier a des questions à poser et des ajustements à faire au niveau de ses connaissances personnelles.

Hernie : « Si tu ne le fais pas, moi je vais te le demander. Ça va augmenter les communications, ça va fatiguer le contrôleur, ça va me fatiguer puis ça va fatiguer tous les autres parce que je prends du temps de fréquence qu'on n'a pas. Mais bon, ils ont leurs contraintes, j'ai mes contraintes, moi ma réalité c'est qu'est-ce que je peux faire pour m'assurer de la sécurité de mon vol. »

Il est pertinent de situer un peu les interactions entre les professions ici : les contrôleurs sont responsables d'une fréquence radio où s'effectuent toutes leurs communications avec les pilotes. Ainsi, si le contrôleur doit aligner 15 avions sur une piste donnée, c'est au moins à 15 pilotes différents qu'il s'adressera. Les communications sont donc structurées pour prendre le moins de temps possible, afin d'éviter que des informations cruciales ne puissent être communiquées. Ici Hernie, un pilote, résout l'ambiguïté primaire en comprenant l'autre, en se mettant à la place du contrôleur lorsqu'il affirme qu'il va demander des informations cruciales à la sécurité de son appareil même s'il sait que le contrôleur aérien a une charge de travail imposante lui aussi. Il admet que cette communication n'est pas sans coût, mais qu'elle doit être établie pour que les connaissances respectives des deux individus impliqués puissent se compléter et se complexifier, évitant ainsi des conséquences et des risques potentiels. Dans ce cas en particulier, le contrôleur accepte de prendre plus de temps pour lui expliquer 
pourquoi il doit prendre une approche qui n'est pas directement en alignement avec la piste d'atterrissage. Nous avons ainsi une situation où la communication n'est pas à prime à bord facile, mais où les deux individus se respectent et font preuve d'ouverture.

\subsubsection{Ambiguïté secondaire résolue}

Les ambiguïtés secondaires résolues incluent des situations où les connaissances sont complexifiées par les perspectives différenciées des parties impliquées. Dans le milieu qui nous intéresse, de telles connaissances synthétisées mènent souvent à des changements dans les procédures mêmes des organisations.

Scott : «Récemment il y a eu un changement dans les routes d'arrivée de l'aéroport de (ville) parce que ça avait été flaggé un peu que les routes de sorties puis les routes d'arrivée, ça se croisait d'une façon bizarre un peu et ça devenait chiant de faire passer le trafic là. Maintenant ça a tout été changé les routes d'arrivées, elles sont différentes. »

Nous avons ici une situation où il y avait des connaissances établies, des routes d'arrivées et de départ d'un aéroport achalandé, qui ont été remises en question sous la forme de flags (drapeaux). Ces items sont là pour signaler un incident potentiel, un élément qui, selon les travailleurs de la sécurité aérienne, peuvent devenir un problème s'ils ne sont pas examinés. Ainsi, face à une connaissance jugée incomplète, c'est-à-dire les procédures de l'aéroport en question, les contrôleurs aériens ont remis en question la façon de faire et ont éventuellement fait en sorte que la procédure a été changée. Ainsi, ils ont complété une connaissance qui avait été établie, mais prouvée incomplète dans l'exercice de leurs fonctions professionnelles. Les perspectives ont été échangées et la procédure améliorée, menant à une diminution directe du risque de conséquences indésirables.

Etienne : « Ça a pris juste un pilote qui l'a dit à quelqu'un puis là on a changé la procédure et on a trouvé quelque chose pour améliorer ça. Mais c'est juste des petites choses de même que ça causait tellement de problèmes quand tu n'es pas au courant de comment ça marche un pilote dans son avion. »

Ici on passe à un commentaire d'un pilote qui a engagé la communication avec les contrôleurs aériens dans le but de les sensibiliser à une connaissance qui était incomplète, la quantité de temps requise pour un pilote de modifier les informations de son FMS (flight information system). Les pilotes, en fonction de leur plan de vol, vont entrer les pistes d'atterrissage dans ce système pour obtenir les autorisations nécessaires lorsque vient l'atterrissage. Lorsque les contrôleurs aériens changent le plan de vol plusieurs fois avant l'atterrissage, les pilotes se retrouvent avec une charge de travail plus élevée lors de l'étape la plus difficile de leur vol, un fait que les contrôleurs aériens ne savaient pas. Un pilote a donc avisé les autorités concernées et les contrôleurs sont maintenant au courant des difficultés engendrées par les changements qu'ils demandent.

\subsubsection{Conséquences/risques}

La colonne conséquences/risques comporte des situations-clés où les participants évoquaient clairement des conséquences de situation où les connaissances n'ont pas été complexifiées.

Roland : «Quand vous faites affaire avec un contrôleur dont la langue maternelle est l'arabe, le tamoul, l'italien ou peu importe et que vous déjà ce n'est pas votre langue maternelle, vous vous parlez chacun dans une deuxième langue. C'est très important de s'en tenir à la phraséologie. Et beaucoup ne le font pas et c'est pour ça qu'ils se mettent dans le trouble. »

Dans ce commentaire, il est question de la phraséologie en vigueur au sein des communications entre les professionnels de l'aviation civile. On parle ici de règles plutôt strictes qui encadrent toutes les communications officielles entre les équipages et les contrôleurs aériens. Ces guides langagiers sont 
issus des millions de communications et des erreurs que celles-ci ont engendrées. Ainsi, les ambiguïtés du genre climb to one three thousand (1300 pieds) et climb two one three thousand (21 300 pieds) sont facilement évitées en utilisant la phraséologie. Roland parle de situations où les gens ne sont pas réceptifs au produit des dialogues organisationnels résultant des ambiguittés secondaires. Ces situations mènent alors à des conséquences néfastes et dans ce cas à des problèmes de communication pouvant conduire à des malentendus sur les altitudes des avions. Les altitudes des avions sont par ailleurs le dernier recours des contrôleurs pour séparer les avions de façon sécuritaire, donc des erreurs de langage peuvent être très coûteuses dans ces moments critiques.

\subsection{Analyses}

Nous avons donc tous les éléments du modèle de Holford (2010) [HOL 10] à partir desquels nous pouvons analyser les différents processus impliqués dans la création de connaissance. Nous pouvons ainsi articuler les mécanismes présents dans le milieu de l'aviation civile à l'aide des relations décrites dans les écrits de Holford. La création de connaissance, si elle est efficace et si elle comprend tous les éléments mentionnés plus tôt, mène à une compréhension plus complète de la situation et qui compte la contribution de plusieurs perspectives. Le résultat est ainsi une perception atténuée de l'occurrence de risque et de conséquences indésirables. De l'autre côté, si les conditions ne sont pas réunies pour un dialogue riche d'opinions variées, on obtient le contraire, c'est-à-dire une connaissance limitée, incomplète et une perception du risque accrue.

En observant le tableau 1, nous sommes en mesure de postuler que l'ambiguïté non-résolue est au cœur des problèmes de communication entre les travailleurs de la sécurité aérienne. En effet, nous avons recensé 141 situations où les interviewés décrivaient des interactions (ou des tentatives d'interaction) où l'ambiguité n'était pas résolue. Nous obtenons donc des situations où l'initiation de la création de connaissance échoue, où l'une des deux entités impliquées dans la conversation n'est pas disponible. Ces situations initiales sont suivies d'une absence de perspectives ajoutées aux connaissances en place et d'un manque de dialogues constructifs. Les échanges de prise de positions contextualisées, telles qu'expliquées dans la section de revue de littérature, ne sont pas partagés. Cet échec de la communication peut être attribuable à l'absence de plusieurs facteurs; le désir de communiquer et de créer des idées, l'ouverture à l'autre et/ou un certain respect mutuel nécessaire à l'écoute. Ces facteurs contribuent au don et à la prise de perspective nécessaires à la résolution de cette ambiguité primaire. Or, dans ces 141 situations, qui représentent $58.5 \%$ de nos commentaires, le dialogue n'est pas possible ni constructif. Dans le milieu de l'aviation, plusieurs hypothèses sont possibles pour expliquer la situation, mais une seule se démarque en ce qui concerne nos résultats. Un constat s'impose alors à travers notre analyse des ambiguités présentes dans le milieu de l'aviation civile .

-les ambiguïtés non-résolues peuvent s'expliquer par les dispositifs mis en place par les organisations de l'aviation civile pour optimiser leurs profits.

Dans notre analyse, nous avons constaté que les pilotes, contrairement aux contrôleurs aériens, ressentent les effets d'être employés par des corporations dont le but premier perçu est la maximisation des profits. Les plans de vol, la quantité de carburant qu'un équipage peut prendre les cost index associés à chaque vol et le nombre de membres de l'équipage : tout est analysé et songé en fonction de réduire les coûts et de maximiser les profits. On parle également de réduction d'effectifs, les compagnies aériennes nord-américaines réduisant de 3 à 2 le nombre de pilotes dans la cabine dans les années 1980. Avec l'automatisation de certains processus, Boeing parle même de la présence potentielle d'un seul pilote en cabine dans ses nouveaux 797 [CNB 19]. Toutes ces mesures ajoutent un stress, une pression sur un travail déjà très complexe.

Il est entendu que la fiabilité demande des ressources importantes et que n'importe quelle organisation veut maximiser sa valeur. Pourtant, naviguer entre profitabilité et fiabilité n'est pas 
évident. Morel (2014) [MOR 14] explique qu'il y a selon lui 2 types de coûts à la fiabilité. Il y a celui de l'investissement en fiabilité, qui inclut tous les coûts des dispositifs qui visent à prévenir les erreurs. Cela comprend les formations que recevront les employés et également tous les coûts liés à la redondance qui doit être mise en œuvre pour réduire la vulnérabilité des opérations. L'auteur évoque également des coûts de non-fiabilité. Ceux-ci comprennent les coûts qui seront engendrés par le manque de fiabilité de l'organisation, donc on parle ici des réparations possibles, de perte de revenus à cause de délais dans les opérations, entre autres. On peut aussi évoquer ici les coûts potentiels que peut causer un accident sur la réputation d'une entreprise et sur la capacité de celle-ci de maintenir son chiffre d'affaire post-accident. Ainsi, le milieu de l'aviation civile semble couper dans les dépenses de fiabilité et par le fait même, augmente potentiellement ses dépenses de non-fiabilité.

Pour donner un exemple de ces mesures de contrôle invasives des coûts, le carburant est souvent limité au strict minimum, plus la quantité nécessaire pour se rendre à l'aéroport de dégagement. Ce dernier est l'aéroport désigné dans le cas où l'aéroport de destination prévu n'est pas disponible ou accessible. Cette quantité de gaz ne permet donc pas d'imprévus et d'extensions au plan de vol, ce qui arrive pourtant tous les jours, réduisant ainsi la marge de manœuvre des pilotes. Ceux-ci se plaignent d'ailleurs de sentiments d'impuissance, où ils n'ont pas leur mot à dire même si c'est leur vie qui est en cause. On obtient des moments où les pilotes sont obligés de se conformer aux directives de l'entreprise et cela dans une situation où l'intervention des pilotes est refoulée ; on parle donc de moments où la complexification des connaissances n'est tout simplement pas possible. Ce commentaire d'un pilote résume bien la situation :

Hernie : « Chaque 100 kilos de carburant doivent être justifiés, pourquoi tu en veux [...] Puis si tu le prends sans l'accord, tu te fais appeler demain matin à $8 \mathrm{~h}$, pourquoi tu as pris 1 tonne de plus ? Si tu dis, bien je devais me sentir à l'aise. Et bien les autres pilotes n'en ont pas besoin, pourquoi toi tu en as besoin ? Puis tu vas devoir justifier et fais-le deux fois et tu vas rentrer dans le bureau. »

On instaure une atmosphère où le partage des connaissances et des ambiguïtés n'est pas encouragé. Dans ce cas-ci, ces ambiguïtés prennent la forme d'une inquiétude liée à la quantité nécessaire de carburant que le pilote doit prendre pour être confortable dans son appareil. Dans le meilleur des cas, cette connaissance serait partagée avec l'organisation et servirait de base à une modification des processus qui diminuerait en fin de compte les risques d'incidents dommageables pour les deux partis.

Autre exemple, il arrive que dans certaines compagnies aériennes, des parties de l'aéronef pourtant cruciales à son maintien soient dysfonctionnelles. Ces avions incomplets volent quand même parce ces composantes de l'avion sont considérées comme indépendantes et ne vont pas influencer trop négativement la performance de l'appareil. L'exemple suivant évoque un incident relié à une dépressurisation effectuée en plein vol.

Fred: «C'est une chaîne d'évènements, c'est l'avion qui était dispatché avec des MEL (Minimum Equipement List) qui faisaient en sorte qu'on a eu un système de pressurisation sur deux qui fonctionnait. »

On voit ici un dispositif de l'organisation, qui porte même un nom officiel (MEL) et qui vise à évaluer le minimum requis pour un avion afin qu'il puisse voler. Dans un milieu où la fiabilité et la sécurité sont aussi capitales, on peut remettre en question les réelles motivations de ces compagnies qui mettent des centaines de vies en danger en agissant ainsi. La préoccupation à l'échec [WEI 08] n'est ici pas assez présente pour que certaines compagnies aériennes soient considérées comme une organisation à haute fiabilité. Les pilotes, de leur côté, doivent composer avec cette situation quotidiennement, ce qui les place dans des situations où la perception du risque est plus élevée.

Ces dispositifs qui visent à sauver de l'argent aux compagnies aériennes mettent souvent des obstacles sur le chemin des pilotes et ne favorisent évidemment pas la communication (C) 2021 ISTE OpenScience - Published by ISTE Ltd. London, UK - openscience.fr 
organisationnelle. Ils ne favorisent pas non plus la remise en question des procédures, étant donné qu'ils ne sont conçus que pour atteindre la profitabilité maximale et ne respectent que cette logique. Les pilotes sont obligés de voler dans ces conditions s'ils veulent conserver leur emploi et c'est la réalité telle qu'ils la connaissent. Il existe bel et bien certaines procédures prévues pour que les pilotes puissent s'exprimer et proposer des améliorations, mais ils n'en font pas beaucoup mention et c'est une infime partie de nos 241 commentaires-clés.

$\mathrm{Si}$ on parle des relations pilotes-contrôleurs aériens, l'exemple de Luc, cité dans la section précédente, nous montre que la relation n'est pas toujours dialectique entre les deux professions. Il arrive parfois que les pilotes n'en fassent qu'à leur tête, et cela, en dépit des recommandations des contrôleurs aériens, ce qui peut mener à des incidents. On peut ici encore formuler l'hypothèse que ces comportements erratiques sont le produit de longues heures de vol et d'impératifs d'efficacité mis en place par les compagnies aériennes.

Eddy: « On lui a demandé ce qu'il voulait, on lui a donné la direction du vent et il a demandé la piste opposée à la direction du vent. La tour de contrôle lui a demandé : est-ce que c'est vraiment cette piste ? Oui. L'envie d'aller à l'hôtel, la journée est finie, on voulait essayer de retourner le plus rapidement possible. Il a préféré, au lieu de faire demi-tour, il a préféré continuer son approche. Il s'est retrouvé face à un (nom du modèle d'avion) qui montait 37000 pieds et avec des vents abattants qui l'ont plaqué au sol, parce qu'il était à très basse altitude et très près de l'aéroport. »

Ici nous avons l'exemple d'un pilote qui n'ouvre pas le dialogue avec le contrôleur aérien. On peut voir l'émancipation de soi du modèle d'Holford, mais pas la recherche de l'identité de l'autre, l'ouverture aux opinions et perspectives complémentaires. Plusieurs pilotes ont l'impression qu'ils ont une énorme charge de travail en raison des nombreux vols de suite qu'ils doivent faire à travers le monde. En effet, les plus petits délais ont des grosses répercussions économiques et dans certaines compagnies, les pilotes doivent justifier tous les retards de plus de 10 minutes aux autorités organisationnelles. Ce faisant, on augmente la pression sur les pilotes à la fin du parcours. C'est une raison pour laquelle ils peuvent être moins réceptifs lors de leurs communications avec les contrôleurs aériens.

Un autre aspect qui peut influencer les conduites organisationnelles des compagnies aériennes est, selon les données que nous avons recueillies, la volonté d'un côté de se déresponsabiliser de toute faute possible et de l'autre d'imputer les défaillances possibles aux pilotes. Le but est ici de se protéger contre les poursuites, qui dans l'aviation civile sont très onéreuses, notamment en raison des sommes exigées en poursuite. On parle également de dommages financiers reliés à l'atteinte à la réputation de l'entreprise en cas d'incidents. Des dispositifs prévus à cette fin sont présents dans le milieu de l'aviation civile et n'ont pas la chance d'être discutés ou du moins soumis à des révisions. Les NOTAM (notice to airmen) sont des fichiers assez volumineux comprenant une vingtaine de pages d'information condensée qu'une équipe de pilotage doit connaître avant le commencement d'un vol [TRA 18]. Ces documents contiennent tous les renseignements pertinents reliés au plan de vol prévu et à toutes les zones que l'équipage va traverser ou est susceptible de croiser en cas d'urgence. Ces informations sont très techniques, difficiles à mémoriser et ne sont pas conçues pour prioriser une information par rapport à une autre, peu importe son importance.

Eddy: «Quand votre urgence va arriver, au milieu de l'atlantique, au milieu du Grand Nord, avec la fatigue, la nuit, le stress, avec un copilote qui n'est pas expérimenté, et que vous allez devoir vous rappeler que la procédure, ah il va falloir faire attention, mais qu'à la page 18 sur 24 des NOTAM, y'a une ligne qui vous affecte, vous allez vraiment me dire que tous les pilotes ont lu les NOTAM de Keflavik en Islande? » 
Les pilotes, ayant pris connaissance de ces NOTAM, sont par le fait de cette lecture tenus responsables par leurs employeurs s'il se passe quelque chose en lien avec le contenu de ces fichiers. Les pilotes, devant arriver à l'aéroport environ $1 \mathrm{~h} 30$ avant le décollage, n'ont que très peu de temps pour intégrer, valider et opérationnaliser cette énorme quantité d'information. Ils n'ont pas le temps de discuter les ambiguïtés qui y sont contenues et aucune rétroaction n'est prévue à cet effet auprès des contrôleurs et des directeurs de vols des compagnies aériennes qui les emploient. Nous sommes ainsi en présence d'une ambiguïté primaire non-résolue, dans une situation qui ne permet pas la complexification des connaissances et qui peut causer des risques et des incidents. La conversation y est à sens unique, et les pilotes ne peuvent y apporter de rétroaction.

Du côté des contrôleurs aériens, nous avons recensé moins d'ambiguïtés primaires en ce qui concerne leurs interactions avec leur employeur. Au contraire, ceux-ci semblent mettre à leur disposition beaucoup de procédures qui sont destinées à améliorer les opérations. On parle ici d'un système de flags qui sont émis pour signaler des potentiels d'incidents et d'une présence managériale qui est disponible dès qu'un incident survient et qui implique les contrôleurs dans ses décisions.

Scott: «Quand il y a un flag qui se lève, [...], il y a une enquête qui est faite pour voir si c'est un événement qui peut se reproduire. Si c'est quelque chose qui peut poser un danger pour la sécurité, c'est sûr qu'il y a une action qui va être prise. Sinon, si c'est quelque chose que toi-même tu remarques, [...] tu le flag à un surveillant, tu vas voir un gestionnaire. »

Le dialogue est ouvert, les deux partis donnent leurs perspectives dans le respect et la reconnaissance des compétences de l'autre. Des recyclages sont également prévus pour discuter des différentes issues que les contrôleurs peuvent rencontrer dans l'exercice de leurs fonctions.

En ce qui concerne la fiabilité, notre analyse a révélé des lacunes au niveau des communications organisationnelles de l'aviation civile, notamment en ce qui concerne les pilotes et les différentes relations dialogiques qu'ils entretiennent dans le cadre de leur profession. Si on considère que des connaissances stagnantes et impersonnelles mènent à une augmentation des risques et des conséquences, peut-on se demander si les compagnies aériennes font réellement partie des organisations à haute fiabilité ?

En effet, les dispositifs mis en place par l'organisation, qui sont dans les cas qui nous concernent implantés pour augmenter l'efficience et la rentabilité des compagnies aériennes, ont un effet négatif sur la complexification des connaissances. Celles-ci n'ont pas l'occasion d'être bonifiées à cause des différentes perspectives des acteurs impliqués et augmentent les risques associés à des connaissances incomplètes et qui ne sont pas contextualisées. On obtient, dans le milieu du transport aérien, plus d'incidents, plus de frustration, plus de conséquences indésirables en général et on rend l'opération de plus en plus névralgique. Comme expliqué dans notre section sur la fiabilité, les opérations de ce genre d'organisations sont déjà excessivement complexes et une petite erreur peut dégénérer en catastrophe en une fraction de seconde.

À la lumière des analyses précédentes, on peut affirmer que les compagnies d'aviation civile ne se comportent pas entièrement comme des organisations à haute fiabilité, même si elles en portent l'étiquette. Si on se rappelle les 5 processus qui contribuent à doter une organisation d'une conscience situationnelle et d'une fiabilité, on constate que ces états d'esprit ne sont pas tous présents. Surtout en ce qui concerne la préoccupation à l'échec, qui évoque une attention de tous les instants pour détecter ce qui n'est pas habituel dans les activités normales de l'entreprise. Il a été vu que les écarts à la normalité font, selon nos données, parties des procédures quotidiennes des compagnies aériennes, qui sont maintenant habituées à travailler avec des équipages diminués et même avec des avions dont toutes les pièces ne sont pas intactes. Par ailleurs, des écarts à la normalité sont parfois signalés par les pilotes à l'organisation, mais celle-ci choisit dans les cas mentionnés de ne pas repartager ces informations avec l'ensemble de ses pilotes afin qu'ils puissent apprendre des incidents des autres. Les 
compagnies aériennes en se protégeant ainsi se rendent très vulnérables, augmentant la probabilité que le même incident se produise et que le pilote réagisse de la même façon, n'ayant pu bénéficier de l'expérience des autres. On peut également affirmer que ces organisations n'ont pas ce que Weick (2008) appelle la réticence à la simplification. Ce processus implique un état de questionnement et de requestionnement perpétuel des éléments de notre environnement et des connaissances qui y sont liées. Nos analyses des sections précédentes démontrent au contraire que la remise en question des procédures organisationnelles n'est pas encouragée et peut se traduire par des interventions négatives de la part de la compagnie. Encore une fois, il est important de noter que ces schémas sont relevés de façon significative par les pilotes, mais pas par les contrôleurs aériens, qui bénéficient d'un encadrement du travail qu'on peut associer aux principes de la fiabilité.

On pourrait même affirmer que certaines compagnies aériennes se comportent selon l'approche de Résilience [SCH 08] et non selon celle de la Prévention. On pourrait s'attendre d'une organisation fiable qu'elle fasse tout ce qui est en son pouvoir pour tenter de limiter le plus possible les risques d'incidents et de se munir des informations nécessaires à la prévention de conséquences indésirables. C'est toutefois le modèle de la résilience qu'on retrouve dans nos données, avec des organisations qui semblent accepter l'inévitabilité des incidents ou à tout le moins qui se satisfont d'opérations qui ne sont pas optimales.

\section{Conclusion}

Notre analyse de données obtenues à partir d'entrevues avec des pilotes et des contrôleurs aériens vise à examiner plusieurs éléments sous un œil nouveau. D'abord, nous avons constaté que les relations dialectiques des professionnels de la sécurité aérienne peuvent être articulées en termes d'ambiguïtés primaires et secondaires, tel qu'avancé par Holford (2010) [HOL 10]. Ces ambiguïtés, qu'elles aient été résolues ou pas, ont un effet important sur la création de connaissances organisationnelles, qui sont opérationnalisées sous la forme de processus et de dispositifs. Ces connaissances, si on parvient à les complexifier et à y amener plusieurs perspectives, contribuent à une diminution de la perception de risque et ainsi à obtenir une meilleure fiabilité et performance dans les opérations quotidiennes des organisations.

Or, nous avons relevé que selon la perception des travailleurs, en particulier les pilotes, les ambiguités primaires et secondaires ne sont pas nécessairement résolues, ce qui occasionne son lot de défis. De plus, nous inférons que ces canaux de communications sont fermés ou incomplets, en partie en raison des dispositifs de maximisation des profits qui sont en place dans les compagnies aériennes. Ceci a un impact subséquent sur la fiabilité générale des opérations, telle que définie dans notre section de littérature.

En termes de limites, ces constats ne sont pas généralisables à l'extérieur des contextes dans lesquels les données ont été recueillies. Nous sommes conscients que les propos que nous avons ne sont pas représentatifs de l'ensemble de la force de travail de l'aviation civile. Un échantillon plus volumineux aurait pu par ailleurs apporter plus de poids à notre argumentation. Il aurait été également pertinent de pouvoir recueillir les opinions de membres de la gestion de compagnies aériennes pour avoir leur version de l'état des connaissances dans l'organisation.

Nous nous permettons ici quelques recommandations, qui découlent de notre compréhension de l'environnement de l'aviation civile et des données que nous avons analysées. Dans le but de réduire cet écart qui semble exister entre le réel des pilotes et les directives des compagnies aériennes, plus de discussions et de recyclages doivent être réalisés entre les pilotes et les autorités qui décident des procédures appliquées. Les circonstances des pilotes doivent être entendues et prises en compte lorsque vient le temps de décider de quelle façon ils peuvent exercer leur métier. Holford (2010) [HOL 10] propose par ailleurs un style de gestion parental (p.154), où les opinions sont partagées dans une atmosphère où chacun peut s'exprimer avec un filet de sécurité, avec immunité. On développe ainsi un 
sentiment de confiance en soi et un environnement où les erreurs ne sont pas condamnées, mais vues comme des opportunités d'apprentissage. Une certaine autonomie est également la bienvenue lorsque vient le temps de créer des connaissances dans un contexte où l'efficience des opérations doit être obtenue sans faire d'erreurs [NON 02]. Selon nous, la clé du succès, dans n'importe quel contexte organisationnel, est le dialogue respectueux et constructif. Après tout, «l'homme est avant tout un homo loquens ; c'est à travers le langage qu'il construit son monde et l'espace humain est d'abord un univers de paroles » [CHA 90, p. 80].

\section{Bibliographie}

[AKT 87] AKTOUF O., Méthodologie des sciences sociales et approche qualitative des organisations une introduction à la démarche classique et une critique. Sainte-Foy: Presses de l'Université du Québec, 1987.

[ANA 07] ANADÓN M., GuILlEMETTE F., La recherche qualitative est-elle nécessairement inductive ? Recherches Qualitatives, Hors Série, 5, 26-37, 2007.

[BEC 01] BECK U., La société du risque : Sur la voie d'une autre modernité, Alto Aubier, Paris, 2001.

[BER 96] Berger P., LuCKMAN T., La construction sociale de la réalité (2e édition., Sociétés). Paris: Méridiens Klincksieck, 1996.

[BOE 96] BOEING., Boeing Forecasts Unprecedented 20-Year Pilot Demand as Operators Face Pilot Supply Challenges. Repéré au : https://boeing.mediaroom.com/2018-07-23-Boeing-Forecasts-Unprecedented-20-Year-Pilot-Demand-asOperators-Face-Pilot-Supply-Challenges, 1996.

[BOH 96] BOHM D., NiCHOL L., On dialogue (Revised and expanded ed.). London: Routledge. Pp.8-9, 1996.

[CHA 90] ChantAla A., BÉDARD R., "La gestion, une affaire de parole”, dans Chanlat, J.F. (Ed.), L'Individu dans l'organisation : les dimensions oubliées, Les Presses de L’Université Laval, Les Éditions ESKA, Québec, pp. 79-99, 1990.

[CHI 13] Chiapello E., Gilbert P., Brault C., Sociologie des outils de gestion : Introduction à l'analyse sociale de l'instrumentation de gestion. (Collection Repères. Grands repères manuels). Paris: La Découverte, 2013.

[CNB 19] CNBC, Some airlines want Boeing's new ' $797^{\prime}$ to fly with just one pilot on board. Disponible sur: https://www.cnbc.com/2019/05/20/boeings-new-797-could-be-built-to-fly-with-just-one-pilot-on-board.html, 2019.

[DEJ 14] DEJOURS C., Le facteur humain (6e édition). Presses Universitaires de France, 2014.

[DEJ 16] Dejours C., L'évaluation du travail à l'épreuve du réel : Critique des fondements de l'évaluation (Sciences en questions). Éditions Quae, 2016.

[DIL 08] DiLlON R.L., TINSLEy C.H., How Near-Misses Influence Decision Making Under Risk: A Missed Opportunity for Learning. Management Science, 54(8), 1425-1440, 2008.

[DUJ 04] DUJARIER M., Le management désincarné : Enquête sur les nouveaux cadres du travail (Poche / Essais). Éditions La Découverte.

[DUR 04] DuRso F.T., DATTELl A., SPAM: The real-time assessment of SA. A cognitive approach to situation awareness: Theory and application, 137-154, 2004.

[ELL 90] ELLUL J., La technique ou L'enjeu du siècle (Classiques des sciences sociales). Paris: Économica, 1990.

[END 01] ENDSLEY M.R., Designing for situation awareness in complex systems. Second International Workshop on Symbiosis of Humans Artifacts and Environment, disponible sur : http://www.satechnologies.com/Papers/pdf/SA\%20design.pdf, 2001.

[FOR 06] FORTIN F., CôTÉ J., FILION F., Fondements et étapes du processus de recherche. Montréal: Chenelièreéducation, 2006.

[FRE 17] FREY C., OSBORNE M., The future of employment: How susceptible are jobs to computerisation? Technological Forecasting \& Social Change, 114(C), 254-280, 2017.

[GEL 13] Gelhausen M., BeRSTER P., WiLKEN D., Do airport capacity constraints have a serious impact on the future development of air traffic? Journal of Air Transport Management, 28, 3-13, 2013.

[GID 84] GIDDENS A., The constitution of society outline of the theory of structuration. Berkeley: University of California Press, 1984. 
[HAl 19] Hallee Y., Garneau J., Deschenaux F., RoYer C., L'abduction comme mode d'inférence et méthode de recherche : De l'origine à aujourd'hui. Recherches Qualitatives, 38(1), 124-140, 2019.

[HOL 09] HOLFORD W.D., Risk, knowledge and contradiction: An alternative and transdiciplinary view as to how risk is induced. Futures, 41(7), 455-467, 2009.

[HOL 10] HOLFORD W.D., Knowledge construction and risk induction/mitigation in dialogical workgroup processes. Qualitative Research in Organizations and Management, 5(2), 127-161, 2010.

[IAT 19] IATA, L'IATA publie les données de 2018 en matière de sécurité aérienne. repéré au : https://www.iata.org/contentassets/928a418fcf4c420fa62e198df1d39335/2019-02-21-01-fr.pdf, 2019.

[IAT 19] IATA, IATA Annual Review. repéré au https://www.iata.org/contentassets/c81222d96c9a4e0bb4ff6ced0126fobb, 2019.

[KAK 02] KaKIHARA M., SØRENSEN C., Exploring Knowledge Emergence: From Chaos to Organizational Knowledge. Journal of Global Information Technology Management, 5(3), 48-66, 2002.

[KLO 01] KLOMAN H.F., Four cubed. Risk Management, 48(9), 23-30, 2001.

[LAP 98] LAPORTE T., CONSOLINI P., Theoretical and operational challenges of "high-reliability organizations": Airtraffic control and aircraft carriers. International Journal of Public Administration, 21(6-8), 847-852, 1998.

[LAW 86] LAWRENCE P.R., LORSCH J.W., dans DUBOIS J-C. (1996), L'analyse du risque, une approche conceptuelle et systémique. Montreal : Chenelière/McGraw-Hill, 1986.

[LEK 11] LEKKa C., SUgDEN C., The successes and challenges of implementing high reliability principles: A case study of a UK oil refinery. Process Safety and Environmental Protection, 89(6), 443-451, 2011.

[MIL 91] Miles M., Huberman A.M., Analyse des données qualitatives recueil de nouvelles méthodes (Pédagogies en développement). Montréal: Éditions du Renouveau pédagogique, 1991.

[MIL 84] Miles M., Huberman A.M., Drawing Valid Meaning from Qualitative Data: Toward a Shared Craft. Educational Researcher, 13(5), 20-30, 1984.

[MIL 18] Milosevic I., Bass A., Combs G., The Paradox of Knowledge Creation in a High-Reliability Organization: A Case Study. Journal of Management, 44(3), 1174-1201, 2018.

[MOR 14] MOREL, C., Les Décisions Absurdes : Tome 2. (Folio Essais), Éditions Gallimard, 2014.

[NON 06] NonAKA, I., Von KRogh G., VoelPel S., Organizational Knowledge Creation Theory: Evolutionary Paths and Future Advances. Organization Studies, 27(8), 1179-1208, 2006.

[NON 95] NONAKA, I., TAKEUCHI H., The knowledge-creating company: How Japanese companies create the dynamics of innovation. New York: Oxford University Press, 1995.

[NON 03] NONAKA, I., TOYAMA R., The knowledge-creating theory revisited: Knowledge creation as a synthesizing process. Knowledge Management Research \& Practice, 1(1), 2-10, 2003.

[NON 05] NONAKA, I., TOYAMA R., The theory of the knowledge-creating firm: Subjectivity, objectivity and synthesis. Industrial and Corporate Change, 14(3), 419-436, 2005.

[NON 02] NONAKA, I., TOYAMA R., A firm as a dialectical being: Towards a dynamic theory of a firm. Industrial and Corporate Change, 11(5), 995-1009, 2002.

[NON 07] NONAKA, I., TOYAMA R., Strategic management as distributed practical wisdom (phronesis). 16(3), 371-394, 2007.

[NON 04] NONAKA, I., TAKEUCHI H., Hitotsubashi on knowledge management. Singapore: John Wiley \& Sons, 2004.

[OAC 18] OACI, Solid passenger traffic growth and moderate air cargo demand in 2018, OACI. Repéré au : https://www.icao.int/Newsroom/Pages/FR/Solid-passenger-traffic-growth-and-moderate-air-cargo-demand-in2018.aspx, 2018.

[PER 03] Perret V., Seville M., Fondements épistémologiques de la recherche, dans Thiétart, R.A. Méthodes de Recherche en Management. Ounod. Paris. Pp.13-33, 2003.

[PER 84] PERROw C., Normal accidents living with high-risk technologies. New York: Basic Books, 1984.

[RAD 18] RADIO-CANADA, Une pénurie mondiale de pilotes de ligne à l'horizon. Repéré au : https://ici.radiocanada.ca/nouvelle/1121960/penurie-pilotes-avion-recrutement-aviation, 2018. 
[ROC 98] Rochling G., LA Porte T., Roberts K., The Self-Designing High-Reliability Organization: Aircraft Carrier Flight Operations at Sea. Naval War College Review, 51(3), 97-113, 1998.

[SAG 04] SAgAn S., The Problem of Redundancy Problem: Why More Nuclear Security Forces May Produce Less Nuclear Security. Risk Analysis, 24(4), 935-946, 2004.

[SAU 18] Saunders B., Sim J., Kingstone T., Baker S., Waterfield J., Bartlam B., Jinks C., Saturation in qualitative research: Exploring its conceptualization and operationalization. Quality \& Quantity, 52(4), 1893-1907, 2018.

[SCH 08] Schulman P.R., RoE E., High reliability management: Operating on the edge. Stanford University Press, 2008.

[Shi 12] Shirali G.H.A., Mohammadfam I., Motamedzade M., Ebrahimipour V., Moghimbeigi A., Assessing resilience engineering based on safety culture and managerial factors. Process Safety Progress, 2012.

[STA 20] Statistique Canada, Mouvements mensuels des aéronefs : principaux aéroports - tours de contrôle et stations d'information de vol de NAV CANADA, repéré au: https://www150.statcan.gc.ca/n1/pub/51-004-x/51-004x2020002-fra.htm, 2020.

[THE 18] The Guardian, Boeing raises prospect of only one pilot in the cockpit of planes. Disponible sur : https://www.theguardian.com/world/2018/feb/09/boeing-raises-prospect-of-only-one-pilot-in-the-cockpit-of-planes, 2018.

[THI 03] THIETART R.A., Méthodes de Recherche en Management. Ounod. Paris, 2003.

[TRA 18] TRANSPORT CANADA, Les NOTAM, document TP 2228F-29, disponible sur: https://www.tc.gc.ca/fra/aviationcivile/publications/tp2228-notam-3527.html, 2018.

[WEI 88] WEICK K.E., Enacted sensemaking in crisis situations. Journal of Management Studies, 25: 305-317, 1988.

[WEI 01] WeICK K.E., Managing the Unexpected. Assuring High Performance in an Age of Complexity, Jossey-Bass, San Francisco, CA, 2001.

[WEI 07] WeICK K.E., SUTCLIFFE K.M., Managing the unexpected: Resilient performance in an age of uncertainty. Second edition. San Francisco: Jossey-Bass, 2007.

[WEI 08] WeICK K.E., SUTCLIFFE K.M., Organizing for high reliability: Processes of collective mindfulness. Crisis Management, 3: 81-123, 2008.

[WIS 93] Wise J., Hopkin V., GibSON R., STAGer P., StUbler W., Verification and Validation of Complex Systems: Human Factors Issues. Proceedings of the Human Factors and Ergonomics Society Annual Meeting, 37(17), 11651169, 1993.

[WRE 06] Wreathall J., Properties of resilient organizations: An initial view. Resilience Engineering: Concepts and Precepts. 258-268, 2006.

[YIN 84] YIN R.K., Case Study Research. Design and Methods, Sage, Thousand Oaks, CA, 1984. 